\title{
Infecciones fúngicas emergentes
}

\author{
Marcia Garnica Marcio Nucci \\ Hospital Universitario, Universidad Federal de Rio de Janeiro, Rio de Janeiro, Brasil
}

\section{Palabras clave}

Pacientes inmunodeprimidos • Infecciones fúngicas emergentes

\section{Resumen}

La epidemiología de las infecciones fúngicas invasivas se encuentra en una fase evolutiva. Han aparecido levaduras distintas de Candida albicans y mohos distintos de Aspergillus fumigatus como causas significativas de micosis invasivas en pacientes gravemente inmunodeprimidos. Mientras que en algunos casos estos cambios pueden estar relacionados con intervenciones médicas, como el uso de agentes antifúngicos profilácticos, en la mayoría de los casos parecen constituir una consecuencia de variaciones en el hospedador. La inmunosupresión más grave o diferentes tipos de inmunosupresión impactan tanto sobre el riesgo como sobre las infecciones que aparecen. Estos factores han alterado la epidemiología de la infección en receptores de trasplantes de órganos, recién nacidos prematuros y pacientes gravemente enfermos. En nuestra revisión se comenta la epidemiología de algunas infecciones fúngicas que han aparecido en los años más recientes, destacando los factores potenciales asociados a su emergencia y las implicaciones prácticas de estos cambios epidemiológicos. Además, se abordan por separado algunas características especiales registradas en niños y en recién nacidos.

Copyright @ 2010 Nestec Ltd., Vevey/S. Karger AG, Basel

\section{Introducción}

La epidemiología de las infecciones fúngicas invasivas ha cambiado durante los últimos 20 años. Su incidencia global ha aumentado y la población de pacientes con riesgo se ha expandido. Actualmente incluye una amplia lista de procesos médicos, como el trasplante de órganos sólidos (TOS) y el trasplante de células madre hematopoyéticas (TCMH), cáncer, tratamiento inmunosupresor, SIDA, parto prematuro, edad avanzada y cirugía importante [1-4]. Además, la etiología de estas infecciones ha experimentado un cambio. En la década de los 80, las levaduras (especialmente, Candida albicans) eran los agentes más frecuentes de las micosis invasivas. En los últimos años, los mohos han llegado a ser más corrientes que las levaduras en ciertos grupos de pacientes, como los receptores de TCMH. En estos pacientes, en quienes la candidiasis todavía es la micosis invasiva más frecuente, las especies distintas de Candida albicans representan más del 50\% de los casos [5]. Entre los mohos, la aspergillosis causada por especies distintas de fumigatus [6], la cigomicosis [7], la fusariosis [8] y la infección causada por mohos negros (feohipomicosis) han sido notificadas crecientemente [9]. Los motivos exactos de estos cambios no se conocen a ciencia cierta. En algunos casos, pueden estar relacionados con intervenciones médicas específicas, como la profilaxis antifúngica [10] y el uso de dispositivos médicos [11-13]. No obstante, en la mayoría de los casos, parecen ser consecuencia de cambios en el hospedador, con inmunodepresión más grave [14].

Dr. Marcio Nucci, Assoc. Prof., Department of Internal Medicine Hematology Service, Hospital Universitário Clementino Fraga Filho Universidade Federal do Rio de Janeiro

Av. Brigadeiro Trompovsky s/n, Rio de Janeiro 21941-590 (Brazil) Fax +55 212562 2460, E-Mail mnucci@ hucff.ufrj.br (c) 2010 Nestec Ltd., Vevey/S. Karger AG, Basel

www.karger.com/ans 
Se dispone de pocos datos con respecto a infecciones fúngicas emergentes, específicamente en niños. Mientras que numerosos aspectos de estas infecciones parecen similares a los de los adultos, los niños y los recién nacidos presentan ciertas diferencias en las manifestaciones epidemiológicas, fisiopatológicas y clínicas [15].

Las infecciones fúngicas emergentes constituyen un problema muy importante para los médicos, dado que conllevan frecuentemente un pronóstico ominoso como consecuencia de diversos factores: los médicos de adultos y los pediatras pueden desconocer la presentación clínica del nuevo patógeno, con la consecuencia de un retraso en el establecimiento del diagnóstico; contar con un laboratorio competente es imperativo para identificar apropiadamente el agente y realizar pruebas de sensibilidad antifúngica, dado que los patógenos emergentes suelen ser resistentes a diversos fármacos antifúngicos. Por último, es más probable que estas infecciones emergentes aparezcan en pacientes gravemente inmunodeprimidos. En esta revisión, comentamos la epidemiología y el diagnóstico de algunas infecciones fúngicas que han aparecido en los últimos años, así como las modificaciones en el hospedador que contribuyen a estas infecciones.

\section{Modificaciones en el hospedador}

Los factores del hospedador son probablemente los determinantes más importantes del surgimiento de infecciones fúngicas causadas por patógenos inusitados. Estos hongos no son habitualmente patógenos en el hospedador normal (e incluso en pacientes con inmunodepresión menos grave), pero aparecen en pacientes con inmunodepresión grave. De hecho, la población de pacientes inmunodeprimidos se está incrementando en diversas situaciones.

En pacientes con cáncer, los cambios en el modo de tratar la enfermedad neoplásica subyacente pueden ejercer una considerable influencia sobre el sistema inmunitario. Por ejemplo, el uso de donantes no emparentados en el TCMH es una opción para varias enfermedades, como la leucemia aguda, la anemia de Fanconi y otras. Este tipo de TCMH se asocia a una mayor incidencia de enfermedad del injerto contra el hospedador (EICH), que resulta en una inmunosupresión acumulada, especialmente en la inmunidad mediada por células T $[16,17]$. En consecuencia, la aspergilosis invasiva (AI) ha llegado a ser una infección emergente en estos pacientes. Además, en el TCMH se están utilizando otras fuentes de células madre, como la sangre del cordón umbilical, cambiando el curso y la duración de la neutropenia en la primera fase del TCMH e incrementando, de este modo, los riesgos de infecciones fúngicas.

En recién nacidos (una población conocida por su elevado riesgo de candidiasis invasiva aguda), se han descrito infecciones causadas por mohos en bebés prematuros que pesan $<1.500 \mathrm{~g}$. Entre los factores de riesgo principales destacan el uso de corticosteroides, la hospitalización prolongada, los fagocitos inmaduros y el traumatismo de la piel. En esta población, la neutropenia no constituye un factor de riesgo importante, tal como se ha demostrado en un estudio en 44 neonatos con AI, en quienes sólo 1 caso presentaba un recuento bajo de neutrófilos [18].

También se ha reportado cada vez más la presencia de AI en pacientes gravemente enfermos, sobre todo en aquéllos con neumopatías obstructivas crónicas tratados con corticosteroides y en pacientes con insuficiencia hepática grave $[19,20]$.

Se han relacionado varios agentes farmacéuticos con el incremento del riesgo de infecciones fúngicas invasivas. Entre ellos destacan los análogos de la purina, fludarabina, cladribina y desoxicoformicina, utilizados en el tratamiento de cánceres hematológicos [21], así como anticuerpos monoclonales. Un ejemplo de estos últimos es el alemtuzumab (Campath), un anticuerpo monoclonal anti-CD52 que produce una intensa depleción de las células $\mathrm{T}$ y se utiliza en neoplasias linforreticulares y con la finalidad de una depleción de células T en el TCMH alogénico, y el infliximab (un antagonista del factor $\alpha$ de necrosis tumoral) en receptores de TOS [21-23]. Los agentes asociados adicionales comprenden el daclizumab (un antagonista del receptor de interleucina 2), utilizado para el tratamiento de la EICH resistente a esteroides en receptores de TCMH alogénico y diversas vasculopatías colagénicas [24-26].

\section{Levaduras emergentes: fungemia}

Tal como se ha mencionado anteriormente, C. albicans ha sido considerado el agente más frecuente de la infección invasiva por levaduras. Con la introducción y el uso generalizado del antimicótico fluconazol, la incidencia global de candidemia ha disminuido, con una reducción de la infección causada por C. albicans y un incremento de las infecciones causadas por Candida glabrata [27]. Desde comienzos de la década de los 90, $C$. 
glabrata ha aparecido como el segundo agente más frecuente de candidemia en Estados Unidos [28]. Además, C. glabrata se ha asociado recientemente a candidiasis orofaríngea en pacientes sometidos a radioterapia por cáncer de cabeza y cuello [29]. Un problema muy importante en la infección causada por C. glabrata es la disminución de su sensibilidad al fluconazol. Con los azoles más recientes, la resistencia cruzada constituye también un problema, dado que el principal mecanismo de resistencia al fluconazol es la sobreexpresión de bombas de eflujo de múltiples fármacos [30]. En un extenso estudio, en el que se evaluaron 610 aislados de C. glabrata obtenidos de pacientes con infecciones invasivas de diferentes partes del mundo, de 46 aislados de C. glabrata resistentes a fluconazol, sólo $13 \%$ fueron sensibles al voriconazol, $4 \%$ al posaconazol y $8,7 \%$ al ravuconazol [31]. En una serie de casos de infecciones intercurrentes en un solo centro, se ha suscitado el problema de la posible aparición de C. glabrata resistente a voriconazol como una amenaza en personas tratadas con este fármaco [32].

En algunas partes del mundo, especialmente en países de América Latina, Candida parapsilosis y Candida tropicalis prevalecen sobre C. glabrata como segunda causa principal de candidemia [33]. Así se describe también en niños, con prevalencias del 24 al 31\% de C. parapsilosis y 16 a $25 \%$ de $C$. tropicallis $[34,35]$. Aunque se desconocen los motivos de la emergencia de estos microorganismos, factores como el cuidado deficiente de catéteres intravenosos, la contaminación del líquido de infusión y la colonización de los trabajadores de salud han sido implicados en las infecciones por C. parapsilosis antes bien que la presión de selección inducida por fármacos [36]. Las diferencias geográficas recalcan la complejidad de la epidemiología. Numerosos factores, incluyendo las especialidades de los centros médicos regionales, los modelos de prácticas y los factores de hospedadores, sustentan probablemente la emergencia de diferentes especies de Candida como patógenos predominantes. Los datos preliminares de pruebas de sensibilidad in vitro con las equinocandinas permiten suponer que algunos aislados de C. parapsilosis presentan concentraciones mínimas inhibidoras superiores a las de otras especies de Candida [37]. Aunque las consecuencias clínicas de estos datos no se conocen, un trabajo reciente sobre el desarrollo de resistencia a múltiples equinocandinas durante la exposición a estos agentes suscita un problema [38], especialmente porque dichos agentes se utilizan cada vez más como tratamiento fundamental de la candidemia.

Infecciones fúngicas emergentes
Candida rugosa ha sido reportada sólo en raras ocasiones como patógeno humano. En 1994 se describió un brote de fungemia causado por este microorganismo en una unidad de quemados de EE.UU. No se identificó ninguna fuente obvia y Dube y cols. [39] asociaron su aparición al uso de nistatina tópica en heridas de quemaduras. Más recientemente se notificó otro brote en una unidad de cuidados intensivos de un hospital de atención terciaria en Brasil durante un estudio de vigilancia de candidemia en 6 centros de 3 ciudades diferentes. Se identificaron 6 casos en el curso de un periodo de 4 meses. Todos los pacientes tenían exposiciones coexistentes que son muy comunes para pacientes afectados de candidemia, como catéteres venosos centrales, uso de antibióticos, cirugía y respiración asistida. Nuevamente, no se identificó ninguna fuente de infección; no obstante, la tipificación del $\mathrm{ADN}$ reveló que todos los aislados estaban genotípicamente relacionados [40]. En un estudio subsiguiente, realizado en la misma unidad, se evaluó prospectivamente a 349 pacientes con cultivos bisemanales de zonas colonizadas con objeto de investigar el valor del pronóstico de la colonización con Candida en el diagnóstico de candidemia [41]. Sorprendentemente, en el 15\% de las 1.400 muestras positivas creció C. rugosa, especie que representaba el $43.7 \%$ de los 32 episodios de candidemia (datos no publicados). En consecuencia, aunque la mayoría de los informes de infección causada por C. rugosa se basaron en el contexto de brotes, se dispone de datos que dan a entender que esta especie puede ser endémica en algunas instituciones.

Especies de Trichosporon han sido asociadas a fungemia e infección diseminada desde finales de la década de los 80. Este microorganismo causa más frecuentemente infección diseminada en pacientes cancerosos neutropénicos [42]. Por el contrario, en un trabajo efectuado recientemente en un único centro oncológico, la mayoría de las infecciones correspondían a fungemias relacionadas con catéteres [43]. Aunque los motivos de estos cambios no son claros, es posible que el empleo generalizado de fluconazol pueda haber desempeñado cierto papel, dado que este agente es activo in vitro frente a especies de Trichosporon [44]. Más recientemente se ha reportado la presencia de tricosporonosis en recién nacidos [45]. La mayoría de los pacientes eran prematuros, con un peso medio al nacer de $<1.000 \mathrm{~g}$. La mayoría de los casos presentaban infección diseminada y en más del $90 \%$ de los casos se aisló el hongo en las vías respiratorias.

Ann Nestlé [Esp] 2009;67:135-142 137 


\section{Otras levaduras emergentes}

Las infecciones criptocócicas aparecen con una distribución casi mundial en hospedadores inmunodeprimidos. Normalmente, las infecciones se deben a Cryptococcus neoformans; no obstante, Cryptococcus gattii causa enfermedad en personas inmunocompetentes de una manera geográficamente restringida, apareciendo la mayoría de los casos en Australia. Un desarrollo notable es la reciente identificación de C. gattii como causa de infección invasiva en animales y personas inmunocompetentes en la isla de Vancouver, Canadá occidental [46, 47]. Desde 1999 se ha identificado un brote que actualmente afecta a $>100$ personas [46]. El microorganismo, que se creyó que sólo prosperaba en regiones tropicales, se recuperó en el medio ambiente en una zona climática templada; el motivo de que apareciese precisamente en esta región no ha sido determinado. Se ha sugerido que factores ambientales, concretamente una tendencia al calentamiento regional, puede apoyar su propagación [47]. Está justificada la realización de más estudios para determinar la importancia clínica de este hongo y definir de qué manera los factores ambientales pueden desempeñar un papel en sustentar la aparición de hongos patógenos.

\section{Mohos emergentes}

La epidemiología de las infecciones por mohos ha cambiado considerablemente en los 10 últimos años. La incidencia de AI ha aumentado significativamente [48] y las infecciones causadas por mohos que presentan resistencia a los agentes antifúngicos convencionales, como especies de Fusarium y los cigomicetos, han sido reportadas cada vez más [49]. La incidencia de AI ha aumentado en un $357 \%$ desde 1980 [50, 51] y en la actualidad supera a la candidiasis invasiva como infección fúngica invasiva más frecuente $[52,53]$.

La mayoría de los casos de AI aparecen en pacientes con neoplasias hematológicas o en receptores de TCMH. En el primero de los grupos, la leucemia mieloide aguda y la leucemia linfocítica aguda de recaída son los procesos subyacentes más frecuentes [50,54]; sin embargo, en los últimos años, la AI se ha diagnosticado crecientemente en otros procesos, como la inmunodeficiencia hereditaria y el TOS [50]. En una encuesta multicéntrica, recientemente publicada, en 139 pacientes pediátricos con AI, estas dos condiciones representan el $23 \%$ de todos los casos de AI. En adultos, la incidencia de AI ha aumentado en pacientes con mieloma. Los datos de un estudio en una población con TCMH revelaron que el riesgo de AI era 4,5 veces superior en pacientes con mieloma múltiple en comparación con pacientes afectados de leucemia mieloide crónica en la fase crónica [6]. El incremento reportado de AI en pacientes con mieloma múltiple puede estar relacionado con cambios en su tratamiento, con la incorporación del concepto de terapia secuencial agresiva con quimioterapia intensiva, autotrasplantes dobles y alotrasplantes no mieloablativos [55].

Recientemente, la edad de los pacientes ha sido considerada un factor de riesgo de AI, con mayor incidencia en pacientes de $>10$ años. Se ha explicado este hecho considerando que la colonización previa es una condición necesaria para el desarrollo de infecciones fúngicas invasivas [56-58].

El momento de la aparición de AI en receptores de TCMH alogénico ha cambiado. Actualmente aparece normalmente en una fase tardía después del TCMH, en pacientes tratados con fármacos inmunosupresores (especialmente dosis elevadas de corticosteroides) para el tratamiento de la EICH, y se diagnostica ocasionalmente en el periodo inicial después del trasplante [6]. Esto es debido a cambios en los procedimientos de trasplante, que han dado como resultado una reducción de la duración de la neutropenia y un incremento de la incidencia y la gravedad de la EICH. Como ejemplos podemos citar los trasplantes con pautas de acondicionamiento de intensidad reducida (trasplante no mieloablativo), trasplantes incompatibles y sin parentesco, infusiones de linfocitos de donante y el uso de anticuerpos monoclonales, como infliximab $[23,25]$. La mayoría de los pacientes pediátricos presentan numerosos factores de inmunodepresión, entre los que destacan la EICH en el $30 \%$, el tratamiento corticosteroideo en el $70 \%$ y la administración de fármacos inmunosupresores (ciclosporina, tacrolimus, daclizumab) en el $43 \%$ de los casos de AI [50]. En una encuesta prospectiva reciente de infecciones fúngicas en niños cancerosos, se registró el $22 \%$ de infecciones fúngicas invasivas en receptores de TCMH. De éstos, el 77\% se observó después de donantes sin parentesco y sólo el 22\% después del TCMH con donantes emparentados. No se observó ningún episodio de infección fúngica invasiva tras el TCMH autólogo [56].

Se ha descrito la linfopenia como factor de riesgo en niños con cáncer y en receptores de TCMH, con presencia en la mayoría de los casos de pacientes no neutropénicos [56]. 
También se ha reportado la aparición tardía de AI en receptores de trasplantes de hígado por una serie de motivos, entre los que destacan una mejor supervivencia en el periodo inicial después del trasplante, la aparición tardía de enfermedad por citomegalovirus y un mayor número de pacientes con disfunción tardía del aloinjerto [59].

Otra tendencia en la AI es la notificación cada vez mayor de infecciones causadas por especies distintas de A. fumigatus. Las especies distintas de fumigatus son más frecuentes en niños que en adultos y representan alrededor del $50 \%$ de los casos $[15,60,61]$. Aspergillus flavus es la especie más frecuente y se asocia particularmente a infección cutánea [50, 60, 62, 63]. Aspergillus terreus suscita una preocupación especial debido a su resistencia in vitro y a su deficiente respuesta clínica a la anfotericina B [44]. A. terreus representaba el 2,1\% de AI en 1996 y el $10,2 \%$ en 2001 en un solo centro [64]. No son evidentes los motivos por los cuales está aumentando la incidencia relativa de infecciones causadas por $A$. terreus, ni tampoco la confirmación de este fenómeno en niños. El pronóstico de la AI causada por $A$. terreus es pobre, con una tasa de mortalidad global del $66 \%$; los pacientes tratados con voriconazol parecen beneficiarse de un mejor resultado [65].

La presentación clínica de la aspergilosis en niños es similar a la registrada en adultos, con afección pulmonar en una gran mayoría de casos. No obstante, el cuadro radiológico puede ser diferente. En un estudio se detectaron cavidades y nódulos en sólo el $25 \%$ de los niños, sin signos de formación de semilunas de aire [66]. En otra serie se demostró que la semiluna de aire y las cavernas se observaban únicamente en niños mayores [67]. La aspergilosis cutánea ha sido reportada más frecuentemente en niños que en adultos, ya sea como único órgano afectado (incidencia del 5 al 14\%) o como parte de la enfermedad diseminada $[50,68]$.

Otra importante diferencia entre adultos y niños en la AI es la actividad del galactomanano sérico como prueba auxiliar para su diagnóstico [69]. El análisis de galactomanano, un inmunoanálisis enzimático sándwich para detectar el antígeno de Aspergillus, está disponible desde 1997. La prueba puede utilizarse como herramienta diagnóstica, aparte de otras características, para seleccionar a los pacientes de alto riesgo (especialmente pacientes con leucemia aguda y receptores de TCMH) o para monitorizar la respuesta al tratamiento [70, 71]. En unos pocos estudios se evalúa el análisis de galactomanano en niños. Mientras que en estudios preliminares se reportaron tasas elevadas de falsos positivos y no se recomendaba la prueba $[15,72]$, en estudios más recientes se demostraron valores pronósticos negativos, similares a los notificados en adultos $[57,73,74]$.

La cigomicosis ha surgido como una infección importante en pacientes gravemente inmunodeprimidos. En una encuesta multicéntrica de infecciones fúngicas en receptores de trasplantes, la proporción de casos de cigomicosis aumentó del 4 al 25\% entre los años 2001 y 2003. Entre los receptores de TCMH se registraron 4 casos de 4.358 trasplantes entre marzo de 2001 y abril de 2002 $(0,09 \%)$, en comparación con 11 casos en 6.660 trasplantes entre mayo de 2002 y junio de 2003 (0,17\%) [75]. El análisis multivariante de factores asociados a la aparición de cigomicosis en comparación con AI reveló que la exposición previa al voriconazol era significativa tanto para el TCMH (cociente de probabilidad: 7,7) como para el TOS (cociente de probabilidad: 10,7). La asociación entre el uso de voriconazol y la cigomicosis está respaldada adicionalmente por 4 informes de cigomicosis intercurrente en 15 pacientes tratados con voriconazol [32, 7678], así como por un estudio monocéntrico de casos y controles en el que se identificó la exposición previa al voriconazol como factor de riesgo significativo de la cigomicosis por análisis multivariante [79]. Aunque el uso del voriconazol podría haber contribuido a la aparición de cigomicosis, otros factores pueden tener la misma importancia. Se dispone de algunos datos indicativos de que la incidencia de cigomicosis estaba aumentando antes de la introducción del voriconazol en la práctica clínica [7]. Entre otros factores probablemente asociados al incremento reciente, destacan la gravedad y el tipo de inmunosupresión. Otra explicación del incremento de la incidencia de cigomicosis es la disminución potencial de la mortalidad relacionada con AI en los años más recientes. Cabe la posibilidad de que el control de una infección muy agresiva haya permitido a los pacientes vivir el tiempo suficiente para presentar una infección subsiguiente con inmunosupresión persistente. Teniendo en cuenta este hecho, la cigomicosis puede considerarse la 'tercera amenaza' de las infecciones fúngicas en receptores de TCMH alogénico.

La mayoría de los casos de cigomicosis en niños aparece en pacientes cancerosos durante la neutropenia, en recién nacidos prematuros o en pacientes con diabetes mellitus. La mortalidad es elevada a pesar del tratamiento antifúngico, con o sin intervención quirúrgica [80].

La fusariosis se ha descrito crecientemente en pacientes inmunodeprimidos. Los datos de un estudio multicéntrico retrospectivo en pacientes con TCMH revelaron 
que el número de casos aumentaba en función del tiempo [8]. El motivo principal de este incremento parece ser la inmunosupresión grave, dado que la incidencia de fusariosis era mayor en trasplantes con donantes incompatibles, y la mayoría de los pacientes presentaban neutropenia grave o EICH. De hecho, un número significativo de casos fueron diagnosticados muy tarde después del trasplante ( $>1$ año) y ninguno de estos pacientes era neutropénico. Esta población de pacientes podría presentar riesgo de fusariosis debido a la grave inmunodeficiencia mediada por células $\mathrm{T}$.

Además de estas infecciones, ocasionalmente ha sido presentada una amplia lista de otros hongos, incluyendo especies de Scedosporium, especies de Acremonium y especies de Phialemonium, como causas de infección en pacientes inmunodeprimidos. En trasplantes de hígado [81] $y$ en receptores de TCMH se ha observado un incremento de la incidencia de infecciones por mohos distintos de Aspergillus [48]. Es probable que este incremento de la incidencia refleje una inmunosupresión más grave. Es interesante destacar que muchos de estos microorganismos parecen presentar cierta restricción geográfica; por ejemplo, las infecciones por Fusarium han sido descritas más frecuentemente en centros médicos del sur de EE.UU. y en Sudamérica [8], mientras que las infecciones por Scedosporium aparecen más frecuentemente en España [82]. Tendrá que aclararse ulteriormente si esto refleja un ses- go en la notificación, la distribución de hospedadores con riesgo o una influencia ambiental sobre la prevalencia fúngica.

\section{Conclusión}

El surgimiento de patógenos fúngicos es una consecuencia de la interacción dinámica entre el hospedador, el entorno y el patógeno. Aunque algunas infecciones fúngicas parecen haber surgido como consecuencia de la presión selectiva del uso generalizado de agentes antifúngicos profilácticos, como es el caso (por lo menos en parte) de la infección causada por C. glabrata, la inmensa mayoría de infecciones fúngicas aparecen debido a una inmunosupresión grave. En consecuencia, dado que se está expandiendo el número y las categorías de pacientes inmunodeprimidos, no es sorprendente que aumente la lista de infecciones fúngicas. Los médicos de adultos y los pediatras deben estar conscientes de la existencia de nuevos patógenos y nuevas entidades y presentaciones clínicas con objeto de establecer un diagnóstico rápido y exacto.

\section{Agradecimiento} CNPq.

\section{Bibliografía}

1 Brown JM: Fungal infections in bone marrow transplant patients. Curr Opin Infect Dis 2004; 17:347-352.

2 Fishman JA: Overview: fungal infections in the transplant patient. Transpl Infect Dis 2002;4(suppl 3):3-11.

3 Kauffman CA: Fungal infections in older adults. Clin Infect Dis 2001;33:550-555.

$\checkmark 4$ Kaufman D: Fungal infection in the very low birthweight infant. Curr Opin Infect Dis 2004;17:253-259.

5 Pappas PG, Rex JH, Lee J, et al: A prospective observational study of candidemia: epidemiology, therapy, and influences on mortality in hospitalized adult and pediatric patients. Clin Infect Dis 2003;37:634-643.

6 Marr KA, Carter RA, Boeckh M, et al: Invasive aspergillosis in allogeneic stem cell transplant recipients: changes in epidemiology and risk factors. Blood 2002;100:43584366.

7 Kauffman CA: Zygomycosis: reemergence of an old pathogen. Clin Infect Dis 2004;39: 588-590.

\footnotetext{
8 Nucci M, Marr KA, Queiroz-Telles F, et al: Fusarium infection in hematopoietic stem cell transplant recipients. Clin Infect Dis 2004;38:1237-1242.

-9 Revankar SG, Patterson JE, Sutton DA, et al: Disseminated phaeohyphomycosis: review of an emerging mycosis. Clin Infect Dis 2002;34:467-476.

10 Snydman DR: Shifting patterns in the epidemiology of nosocomial Candida infections. Chest 2003; 123:500S-503S.

11 Hsueh PR, Teng LJ, Ho SW, Luh KT: Catheter-related sepsis due to Rhodotorula glutinis. J Clin Microbiol 2003;41:857-859.

12 Reinhardt JF, Ruane PJ, Walker LJ, George WL: Intravenous catheter-associated fungemia due to Candida rugosa. J Clin Microbiol 1985;22:1056-1057.

13 Yamada S, Maruoka T, Nagai K, et al: Catheter-related infections by Hansenula anomala in children. Scand J Infect Dis 1995;27: 85-87.

4 Procop GW, Roberts GD: Emerging fungal diseases: the importance of the host. Clin Lab Med 2004;24:691-719.

15 Steinbach WJ: Pediatric aspergillosis: disease and treatment differences in children. Pediatr Infect Dis J 2005;24:358-364.

- 16 Schutt P, Brandhorst D, Stellberg W, et al: Immune parameters in multiple myeloma patients: influence of treatment and correlation with opportunistic infections. Leuk Lymphoma 2006;47:1570-1582.

17 Wingard JR: Opportunistic infections after blood and marrow transplantation. Transpl Infect Dis 1999;1:3-20.

18 Groll AH, Jaeger G, Allendorf A, et al: Invasive pulmonary aspergillosis in a critically ill neonate: case report and review of invasive aspergillosis during the first 3 months of life. Clin Infect Dis 1998;27:437-452.

19 Bulpa P, Dive A, Sibille Y: Invasive pulmonary aspergillosis in patients with chronic obstructive pulmonary disease. Eur Respir J 2007;30:782-800.
} 
20 Meersseman W, Lagrou K, Maertens J, Van Wijngaerden E: Invasive aspergillosis in the intensive care unit. Clin Infect Dis 2007;45: 205-216.

-21 Anaissie EJ, Kontoyiannis DP, O’Brien S, et al: Infections in patients with chronic lymphocytic leukemia treated with fludarabine. Ann Intern Med 1998;129:559-566.

22 Keating MJ, Flinn I, Jain V, et al: Therapeutic role of alemtuzumab (Campath-1H) in patients who have failed fludarabine: results of a large international study. Blood 2002;99: 3554-3561.

23 Nath DS, Kandaswamy R, Gruessner R, et al: Fungal infections in transplant recipients receiving alemtuzumab. Transplant Proc 2005;37:934-936.

24 Labbe AC, Su SH, Laverdiere M, et al: High incidence of invasive aspergillosis associated with intestinal graft-versus-host disease following nonmyeloablative transplantation. Biol Blood Marrow Transplant 2007; 13:1192-1200.

25 Marty FM, Lee SJ, Fahey MM, et al: Infliximab use in patients with severe graft-versus-host disease and other emerging risk factors of non-Candida invasive fungal infections in allogeneic hematopoietic stem cell transplant recipients: a cohort study. Blood 2003;102:2768-2776.

-26 Tsiodras S, Samonis G, Boumpas DT, Kontoyiannis DP: Fungal infections complicating tumor necrosis factor alpha blockade therapy. Mayo Clin Proc 2008;83:181-194.

- 27 Trick WE, Fridkin SK, Edwards JR, et al: Secular trend of hospital-acquired candidemia among intensive care unit patients in the United States during 1989-1999. Clin Infect Dis 2002;35:627-630.

28 Pfaller MA, Diekema DJ: Twelve years of fluconazole in clinical practice: global trends in species distribution and fluconazole susceptibility of bloodstream isolates of Candida. Clin Microbiol Infect 2004; 10(suppl 1):1123.

-29 Redding SW, Dahiya MC, Kirkpatrick WR, et al: Candida glabrata is an emerging cause of oropharyngeal candidiasis in patients receiving radiation for head and neck cancer. Oral Surg Oral Med Oral Pathol Oral Radiol Endod 2004;97:47-52.

-30 Bennett JE, Izumikawa K, Marr KA: Mechanism of increased fluconazole resistance in Candida glabrata during prophylaxis. Antimicrob Agents Chemother 2004;48:17731777.

31 Pfaller MA, Messer SA, Boyken L, et al: Geographic variation in the susceptibilities of invasive isolates of Candida glabrata to seven systemically active antifungal agents: a global assessment from the ARTEMIS Antifungal Surveillance Program conducted in 2001 and 2002. J Clin Microbiol 2004;42: 3142-3146.
32 Imhof A, Balajee SA, Fredricks DN, et al: Breakthrough fungal infections in stem cell transplant recipients receiving voriconazole. Clin Infect Dis 2004;39:743-746.

33 Colombo AL, Nucci M, Salomao R, et al: High rate of non-albicans candidemia in Brazilian tertiary care hospitals. Diagn Microbiol Infect Dis 1999;34:281-286.

34 Pasqualotto AC, de Moraes AB, Zanini RR, Severo LC: Analysis of independent risk factors for death among pediatric patients with candidemia and a central venous catheter in place. Infect Control Hosp Epidemiol 2007; 28:799-804.

35 Stamos JK, Rowley AH: Candidemia in a pediatric population. Clin Infect Dis 1995;20: 571-575.

36 Pfaller MA: Nosocomial candidiasis: emerging species, reservoirs, and modes of transmission. Clin Infect Dis 1996;22(suppl 2):S89-S94.

37 Pfaller MA, Diekema DJ, Jones RN, et al: Trends in antifungal susceptibility of Candida spp. isolated from pediatric and adult patients with bloodstream infections: SENTRY Antimicrobial Surveillance Program, 1997 to 2000. J Clin Microbiol 2002;40:852856.

38 Moudgal V, Little T, Boikov D, Vazquez JA: Multiechinocandin- and multiazole-resistant Candida parapsilosis isolates serially obtained during therapy for prosthetic valve endocarditis. Antimicrob Agents Chemother 2005;49:767-769.

39 Dube MP, Heseltine PN, Rinaldi MG, et al: Fungemia and colonization with nystatinresistant Candida rugosa in a burn unit. Clin Infect Dis 1994;18:77-82.

40 Colombo AL, Melo AS, Crespo Rosas RF, et al: Outbreak of Candida rugosa candidemia: an emerging pathogen that may be refractory to amphotericin B therapy. Diagn Microbiol Infect Dis 2003;46:253-257.

41 Rosas R, Nucci M, Castelo A, et al: Predictive value of Candida spp. colonization in the diagnosis of candidemia in intensive care unit patients (abstract M-269). Program and Abstracts 44th Interscience Conference on Antimicrobial Agents and Chemotherapy (Washington). Washington, American Society for Microbiology, 2004, p 410.

42 Walsh TJ, Newman KR, Moody M, et al: Trichosporonosis in patients with neoplastic disease. Medicine (Baltimore) 1986;65:268279.

43 Kontoyiannis DP, Torres HA, Chagua M, et al: Trichosporonosis in a tertiary care cancer center: risk factors, changing spectrum and determinants of outcome. Scand J Infect Dis 2004;36:564-569.

44 Pfaller MA, Diekema DJ: Rare and emerging opportunistic fungal pathogens: concern for resistance beyond Candida albicans and Aspergillus fumigatus. J Clin Microbiol 2004; 42:4419-4431.
45 Salazar GE, Campbell JR: Trichosporonosis, an unusual fungal infection in neonates. Pediatr Infect Dis J 2002;21:161-165.

46 Hoang LM, Maguire JA, Doyle P, et al: Cryptococcus neoformans infections at Vancouver Hospital and Health Sciences Centre (1997-2002): epidemiology, microbiology and histopathology. J Med Microbiol 2004; 53:935-940.

47 Kidd SE, Hagen F, Tscharke RL, et al: A rare genotype of Cryptococcus gattii caused the cryptococcosis outbreak on Vancouver Island (British Columbia, Canada). Proc Natl Acad Sci USA 2004;101:17258-17263.

48 Marr KA, Carter RA, Crippa F, et al: Epidemiology and outcome of mould infections in hematopoietic stem cell transplant recipients. Clin Infect Dis 2002;34:909-917.

49 Nucci M: Emerging moulds: Fusarium, Scedosporium and Zygomycetes in transplant recipients. Curr Opin Infect Dis 2003;16: 607-612.

50 Burgos A, Zaoutis TE, Dvorak CC, et al: Pediatric invasive aspergillosis: a multicenter retrospective analysis of 139 contemporary cases. Pediatrics 2008;121:e1286-e1294.

51 McNeil MM, Nash SL, Hajjeh RA, et al: Trends in mortality due to invasive mycotic diseases in the United States, 1980-1997. Clin Infect Dis 2001;33:641-647.

52 Groll AH, Shah PM, Mentzel C, et al: Trends in the postmortem epidemiology of invasive fungal infections at a university hospital. J Infect 1996;33:23-32.

53 van Burik JH, Leisenring W, Myerson D, et al: The effect of prophylactic fluconazole on the clinical spectrum of fungal diseases in bone marrow transplant recipients with special attention to hepatic candidiasis. An autopsy study of 355 patients. Medicine (Baltimore) 1998;77:246-254.

54 Cornet M, Fleury L, Maslo C, et al: Epidemiology of invasive aspergillosis in France: a six-year multicentric survey in the Greater Paris area. J Hosp Infect 2002;51:288-296.

55 Barlogie B, Shaughnessy J, Tricot G, et al: Treatment of multiple myeloma. Blood 2004;103:20-32.

56 Castagnola E, Cesaro S, Giacchino M, et al: Fungal infections in children with cancer: a prospective, multicenter surveillance study. Pediatr Infect Dis J 2006;25:634-639.

57 Castagnola E, Faraci M, Moroni C, et al: Invasive mycoses in children receiving hemopoietic SCT. Bone Marrow Transplant 2008; 41(suppl 2):S107-S111.

58 Dvorak CC, Steinbach WJ, Brown JM, Agarwal R: Risks and outcomes of invasive fungal infections in pediatric patients undergoing allogeneic hematopoietic cell transplantation. Bone Marrow Transplant 2005;36:621629. 
59 Singh N, Avery RK, Munoz P, et al: Trends in risk profiles for and mortality associated with invasive aspergillosis among liver transplant recipients. Clin Infect Dis 2003; 36:46-52.

60 Herbrecht R, Auvrignon A, Andres E, et al: Efficacy of amphotericin B lipid complex in the treatment of invasive fungal infections in immunosuppressed paediatric patients. Eur J Clin Microbiol Infect Dis 2001;20:7782.

61 Walsh TJ, Lutsar I, Driscoll T, et al: Voriconazole in the treatment of aspergillosis, scedosporiosis and other invasive fungal infections in children. Pediatr Infect Dis J 2002; 21:240-248.

62 Abbasi S, Shenep JL, Hughes WT, Flynn PM: Aspergillosis in children with cancer: a 34year experience. Clin Infect Dis 1999;29: 1210-1219.

63 Walmsley S, Devi S, King S, et al: Invasive Aspergillus infections in a pediatric hospital: a ten-year review. Pediatr Infect Dis J 1993;12:673-682.

64 Baddley JW, Pappas PG, Smith AC, Moser SA: Epidemiology of Aspergillus terreus at a university hospital. J Clin Microbiol 2003; 41:5525-5529.

-65 Steinbach WJ, Benjamin DK Jr, Kontoyiannis DP, et al: Infections due to Aspergillus terreus: a multicenter retrospective analysis of 83 cases. Clin Infect Dis 2004;39:192-198.

66 Thomas KE, Owens CM, Veys PA, et al: The radiological spectrum of invasive aspergillosis in children: a 10 -year review. Pediatr Radiol 2003;33:453-460.

67 Taccone A, Occhi M, Garaventa A, et al: CT of invasive pulmonary aspergillosis in children with cancer. Pediatr Radiol 1993;23. 177-180.

68 Vonberg RP, Gastmeier P: Nosocomial aspergillosis in outbreak settings. J Hosp Infect 2006;63:246-254
69 De Pauw B, Walsh TJ, Donnelly JP, et al: Revised definitions of invasive fungal disease from the European Organization for Research and Treatment of Cancer/Invasive Fungal Infections Cooperative Group and the National Institute of Allergy and Infectious Diseases Mycoses Study Group (EORTC/MSG) Consensus Group. Clin Infect Dis 2008;46:1813-1821.

70 Miceli MH, Grazziutti ML, Woods G, et al: Strong correlation between serum aspergillus galactomannan index and outcome of aspergillosis in patients with hematological cancer: clinical and research implications. Clin Infect Dis 2008;46:1412-1422.

71 Woods G, Miceli MH, Grazziutti ML, et al: Serum Aspergillus galactomannan antigen values strongly correlate with outcome of invasive aspergillosis: a study of 56 patients with hematologic cancer. Cancer 2007;110: 830-834.

72 Sulahian A, Tabouret M, Ribaud P, et al: Comparison of an enzyme immunoassay and latex agglutination test for detection of galactomannan in the diagnosis of invasive aspergillosis. Eur J Clin Microbiol Infect Dis 1996;15:139-145

73 Steinbach WJ, Addison RM, McLaughlin L, et al: Prospective Aspergillus galactomannan antigen testing in pediatric hematopoietic stem cell transplant recipients. Pediatr Infect Dis J 2007;26:558-564.

74 Sulahian A, Boutboul F, Ribaud P, et al: Value of antigen detection using an enzyme immunoassay in the diagnosis and prediction of invasive aspergillosis in two adult and pediatric hematology units during a 4-year prospective study. Cancer 2001;91:311-318.
75 Park BJ, Kontoyiannis DP, Pappas PG, et al: Comparison of zygomycosis and fusariosis to invasive aspergillosis among transplant recipients reporting to TRANSNET (abstract M-666). Program and Abstracts 44th Interscience Conference on Antimicrobial Agents and Chemotherapy (Washington), Washington, American Society for Microbiology, 2004, p 411.

76 Kobayashi K, Kami M, Murashige N, et al Breakthrough zygomycosis during voriconazole treatment for invasive aspergillosis. Haematologica 2004;89:ECR42.

77 Marty FM, Cosimi LA, Baden LR: Breakthrough zygomycosis after voriconazole treatment in recipients of hematopoietic stem-cell transplants. N Engl J Med 2004; 350:950-952.

-78 Siwek GT, Dodgson KJ, Magalhaes-Silverman M, et al: Invasive zygomycosis in hematopoietic stem cell transplant recipients receiving voriconazole prophylaxis. Clin Infect Dis 2004;39:584-587.

79 Kontoyiannis DP, Lionakis MS, Lewis RE, et al: Zygomycosis in a tertiary-care cancer center in the era of Aspergillus-active antifungal therapy: a case-control observational study of 27 recent cases. J Infect Dis 2005; 191:1350-1360.

80 Zaoutis TE, Roilides E, Chiou CC, et al: Zygomycosis in children: a systematic review and analysis of reported cases. Pediatr Infect Dis J 2007;26:723-727.

81 Husain S, Alexander BD, Munoz P, et al: Opportunistic mycelial fungal infections in organ transplant recipients: emerging importance of non-Aspergillus mycelial fungi. Clin Infect Dis 2003;37:221-229.

-82 Bouza E, Munoz P: Invasive infections caused by Blastoschizomyces capitatus and Scedosporium spp. Clin Microbiol Infect 2004;10(suppl 1):76-85. 